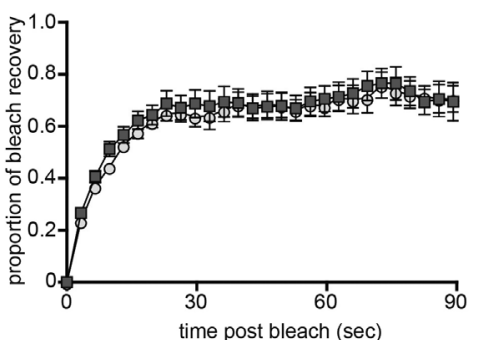

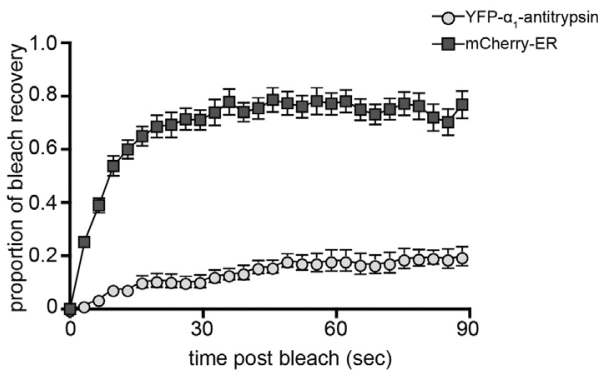
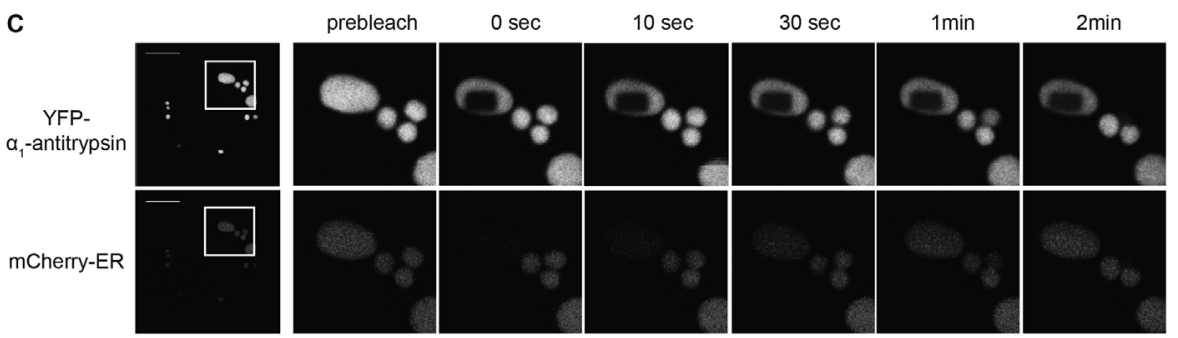

Abstract S126 Figure 1 Assessing ER chaperone mobility in cells expressing wild-type and polymerogenic AAT using 2-colour FRAP. Cells expressing WT (A) or polymerogenic $Z$ (B) AAT were subjected to 2-colour FRAP. Note the ER protein ER-mCherry remains mobile even in the presence of immobile Z-AAT. (C) 2-colour bleaching of a single ER inclusion containing Z-AAT confirms immobility of Z-AAT but free movement of ER-mCherry through the immobile AAT lattice. (AAT; al-antitrypsin)

understanding of the consequences of Z-alpha-1-antitrypsin expression on cellular dysfunction.

Methods We created YFP tagged wild-type and Z-alpha-1-antitrypsin constructs and expressed them in a cell model along with other fluorescently tagged proteins of interest. Using live-cell imaging including photobleaching techniques, we assessed the relative mobilities of alpha-1-antitrypsin and other ER resident proteins. We further assessed the nature of inter-inclusion protein trafficking by creating a permeabilised cell system in which the cytosol could be manipulated or removed.

Results We have shown that inclusions are translationally active ER fragments in which polymerisation occurs. Using fluorescence recovery after photobleaching (FRAP), we observed that despite inclusions containing immobile polymeric alpha-1-antitrypsin, small ER resident proteins including the ER chaperone BiP are able to diffuse freely within them (Figure 1). We observed that inclusions are physically separated from the tubular ER network but despite this, cargo is transported between inclusions in a cytosol-dependent fashion that is dependent on vesicular trafficking components and may involve the ER-Golgi intermediate compartment (ERGIC).

Conclusions We propose that protein movement between physically separated ER inclusions via ER-ERGIC recycling acts to minimise ER heterogeneity in Z-alpha-1-antitrypsin expressing cells. This may reduce the toxic effects of polymer accumulation via increased availability of, for example, ER chaperones within inclusions and is consistent with in vivo observations of a striking lack of toxicity in cells expressing Z-alpha-1-antitrypsin.

\section{S127 GENE THERAPY FOR ALPHA-1-ANTITRYPSIN DEFICIENCY USING A PSEUDOTYPED LENTIVIRUS VECTOR}

${ }^{1} \mathrm{MC}$ Paul-Smith, ${ }^{2} \mathrm{JF}$ Gelinas, ${ }^{1} \mathrm{~K}$ Pytel, ${ }^{1} \mathrm{M}$ Chan, ${ }^{1} \mathrm{C}$ Meng, 'L Cammack, 'L Cameron, ${ }^{1} \mathrm{C}$ Moran, ${ }^{2}$ I Pringle, ${ }^{2} \mathrm{~L}$ Davies, ${ }^{3} \mathrm{M}$ Inoue, ${ }^{3} \mathrm{M}$ Hasegawa, ${ }^{2} \mathrm{SC}$ Hyde, ${ }^{2} \mathrm{DR}$ Gill, ${ }^{1}$ EWFW Alton, ${ }^{1} \mathrm{U}$ Griesenbach. ${ }^{1}$ Imperial College, London, UK; ${ }^{2}$ University of Oxford, Oxford, UK; ${ }^{3}$ DNAVEC, Tsukuba, Japan
Introduction and objectives A protease/anti-protease imbalance is a characteristic feature of inflammatory lung diseases such as cystic fibrosis (CF) and alpha-1-antitrypsin deficiency related emphysema. A recent trial of alpha-1-antitrypsin (hAAT) enzyme replacement therapy (ERT) suggested that hAAT can slow the progression of lung density loss in alpha-1-antitrypsin deficiency (Chapman et al, Lancet 2015). However, the results are modest and ERT is expensive, so gene therapy may be a more appropriate treatment strategy.

The UK Cystic Fibrosis Gene Therapy Consortium has pseudotyped a simian immunodeficiency viral vector with the Sendai virus $\mathrm{F}$ and $\mathrm{HN}$ proteins (rSIV. F/HN) for efficient transduction of airway epithelial cells.

Results Mice were transduced with rSIV. F/HN-hAAT $(1.4 \mathrm{e} 8$ TU/mouse) by nasal instillation and culled 10 days post-transduction. hAAT levels in lung tissue homogenate and epithelial lining fluid (ELF) were 3 logs above controls $(\mathrm{p}<0.05)$, and hAAT concentration in ELF was $92 \pm 28 \mu \mathrm{g} / \mathrm{ml}$, similar to the therapeutic hAAT level in ELF of $70 \mu \mathrm{g} / \mathrm{ml}$ (Figure 1). For comparison, transfection of mouse lung with cationic lipid GL67A, used in the recent Phase IIb trial of non-viral gene therapy for cystic fibrosis, complexed to plasmids carrying hAAT only led to $0.4 \pm$ $0.1 \mu \mathrm{g} / \mathrm{ml}$ in ELF.

A neutrophil elastase (NE) activity assay showed that the recombinant hAAT successfully neutralised NE activity $(\mathrm{p}<$ $0.05)$. In a separate experiment, mice were treated with a single dose of rSIV. F/HN-hAAT (4e7 TU/mouse) and quantification of hAAT one year post-transduction showed that expression was stable over this period. Here, we also demonstrate for the first time that $\mathrm{rSIV}$. F/HN transduction of lung generates significant $(\mathrm{p}<0.05)$ levels of recombinant hAAT protein in serum.

Conclusion In conclusion, rSIV. F/HN produces therapeutically relevant and long-lasting levels of hAAT in murine lung and may offer advantages over enzyme replacement therapy. In addition, we showed that hAAT escapes from the lung into the circulation which may be relevant for a range of diseases including diabetes and graft vs. host disease. 


\section{REFERENCE}

1 Chapman KR, Burdon JG, Piitulainen E et al. Intravenous augmentation treatment and lung density in severe $\alpha 1$ antitrypsin deficiency (RAPID): a randomised, double-blind, placebo-controlled trial. Lancet. 2015;386:360-8

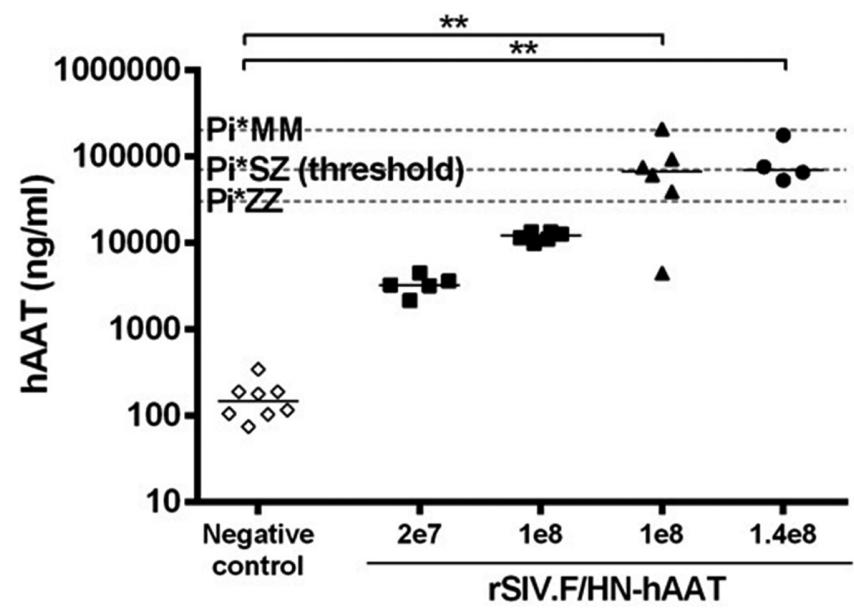

Abstract S127 Figure 1 Expression of hAAT in epithelial lining fluid following treatment with rSIV. F/HN-hAAT. Mice were given between 2e7 and 1.4e8 TU virus and sacrificed 7-10 days post-treatment

\section{S128 SOLUBLE ADAM33 CAUSES AIRWAY REMODELLING TO PROMOTE ALLERGIC AIRWAY INFLAMMATION}

${ }^{1}$ ER Davies, ${ }^{2} \mathrm{JA}$ Whitsett, ${ }^{1} \mathrm{DE}$ Davies, ${ }^{1} \mathrm{HM}$ Haitchi. 'University of Southampton, Southampton, UK; ${ }^{2}$ Cincinnati Children's Hospital Medical Center, Cincinnati, USA

\subsection{6/thoraxjnl-2015-207770.134}

Introduction and objectives $A D A M 33$ is an asthma susceptibility gene associated with bronchial hyperresponsiveness (BHR). It encodes a membrane-anchored protein with metalloprotease (MP) activity whose ectodomain can be shed from the cell surface as a soluble protein (sADAM33-MP). sADAM33-MP levels are increased in asthmatic airways and inversely correlated with FEV1. We have previously generated a pulmonary epitheliumspecific, doxycycline (DOX)-inducible double transgenic (dTg) mouse expressing human (h)sADAM33-MP and found that the transgene caused airway remodelling in the absence of inflammation or BHR. Therefore, as asthma involves gene-environment interactions, we postulated that there is a synergistic relationship between ADAM33-remodelled airways and responses to the common aeroallergen, house dust mite (HDM).

Methods DOX was administered to dTg mice to induce hsADAM33 expression and airway remodelling for up to 6 weeks; single transgenic (sTg) littermate controls were similarly treated. Mice were then sensitised to HDM and challenged with HDM or saline. Airway resistance was measured in response to increasing concentrations of methacholine using the forced oscillation technique in anesthetised mice. Inflammatory cell counts were performed on bronchoalveolar lavage fluid (BALF) and indices of inflammation measured by RTqPCR and Luminex ELISA.

Results We first performed a concentration-response experiment with HDM extract with a standard sensitisation protocol to determine the amount of HDM extract $(6.25 \mu \mathrm{g})$, which elicited minimal BHR and eosinophilia. This low-dose allergen challenge protocol was then applied to dTg Ccsp/ADAM33 and sTg control mice. Allergen challenge of $\mathrm{dTg}$ mice resulted in a significant increase in methacholine-induced airway resistance and eosinophilic airway inflammation compared to HDM-challenged
sTg controls. The dTg mice also showed a significant increase in airway inflammatory mediators IL-5, IL-13 and eotaxin, in addition to markers of remodelling.

Conclusions This study demonstrates that hsADAM33-MP driven airway remodelling enhances susceptibility to HDM with increases in BHR and inflammation. These functional studies demonstrate, for the first time, a gene-environment interaction involving ADAM33 to cause remodelling and the disproportional inflammatory responses seen in the asthmatic airway. sADAM33 might be a potential target for novel disease-modifying therapies.

\section{S129 A TWO SPECIES PROTEOMICS APPROACH TO DETERMINE MMP-12 SUBSTRATES IN COPD}

${ }^{1} \mathrm{~B}$ Mallia-Milanes, ${ }^{2} \mathrm{~A}$ Dufour, ${ }^{1} \mathrm{H}$ Bailey, ${ }^{1} \mathrm{G}$ Meakin, ${ }^{3} \mathrm{~A}$ Leme, ${ }^{1} \mathrm{C}$ Bolton, ${ }^{3} \mathrm{~S}$ Shapiro, ${ }^{2} \mathrm{C}$ Overall, ${ }^{1} \mathrm{~S}$ Johnson. ${ }^{1}$ University of Nottingham, Nottingham, UK; ${ }^{2}$ University of British Columbia, Vancouver, Canada; ${ }^{3}$ University of Pittsburgh, Pittsburgh, USA

\subsection{6/thoraxjnl-2015-207770.135}

Background Genetic variability in MMP-12 is associated with COPD; the matrix Metalloproteinase (MMP)-12 knockout (KO) mouse is resistant to emphysema despite cigarette smoke exposure, strongly implicating MMP-12 in COPD pathogenesis. However, the complete MMP-12 substrate profile (degradome) in COPD remains unknown. Terminal amine isobaric labelling of substrates (TAILS) is a novel proteomic technique allowing identification of a protease degradome on an organism-wide scale. Identification of the MMP-12 degradome will lead to novel drugs, desperately needed in COPD.

Objectives To identify the MMP- 12 degradome in COPD by comparing cigarette smoke exposed MMP-12 KO and wildtype (WT) controls by TAILS and validating these targets against the human COPD sputum proteome during exacerbations and stable disease.

Methods C57BL/6J MMP-12 KO and WT mice $(\mathrm{n}=4)$ were exposed to cigarette smoke and airways sampled by bronchoalveolar lavage (BAL). BAL fluid was analysed by TAILS, high performance liquid chromatography (HPLC) and tandem mass spectrometry (MS/MS). Matched COPD exacerbation and stable disease sputum samples $(n=9)$ were analysed by TAILS, HPLC and MS/MS.

Results The following new MMP-12 targets in the COPD mouse model were identified: alpha-2-HS glycoprotein, anti-thrombin III, clusterin, complement C3, complement C4b, complement factor H-related protein-1, hemopexin, serotransferrin and serum albumin, alpha-2-macroglobulin, beta-1, 4-galactosyltransferase 2, transmembrane protease 7 , DEP domain-containing mTOR-interacting protein, kininogen-1, tumour necrosis factor ligand superfamily member 11 . Of these, alpha-2-HS-glycoprotein, anti-thrombin III, complement factors $\mathrm{C} 3$ and $\mathrm{C} 4 \mathrm{~B}$, hemopexin and serum albumin were identified in both exacerbation and stable COPD human sputum. Furthermore, 1,116 peptides were identified in COPD exacerbation and stable disease sputum, grouped into the following categories: cell adhesion/migration, complement system, acute phase response, extracellular matrix structure/function, anti-microbicidal activity, cytoskeletal function/remodelling, carbohydrate metabolism, oxidoreductase activity, cell death regulation/DNA synthesis/repair, immune response, protease activity, protease inhibition and ATP synthesis/function.

Conclusion This study identifies the MMP-12 degradome in COPD and provides the most comprehensive analysis of the 